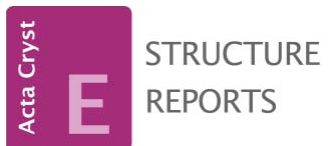

ISSN 1600-5368

\section{Crystal structure of 3-\{1-[(1-allyl-1H- indazol-6-yl)amino]ethylidene\}-6- methyl-2H-pyran-2,4(3H)-dione}

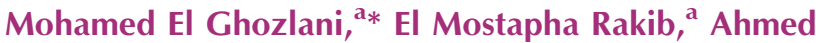 \\ Gamouh, ${ }^{a}$ Mohamed Saadi ${ }^{\mathrm{b}}$ and Lahcen El Ammari ${ }^{\mathrm{b}}$ \\ a'Laboratoire de Chimie Organique et Analytique, Université Sultan Moulay Slimane, \\ Faculté des Sciences et Techniques, Béni-Mellal, BP 523, Morocco, and ${ }^{\mathbf{b}}$ Laboratoire \\ de Chimie du Solide Appliquée, Faculté des Sciences, Université Mohammed \\ V-Agdal, Avenue Ibn Battouta, BP 1014, Rabat, Morocco. *Correspondence e-mail: \\ m_elghozlani@yahoo.fr
}

Received 6 November 2014; accepted 7 November 2014

Edited by W. T. A. Harrison, University of Aberdeen, Scotland

In the title compound, $\mathrm{C}_{18} \mathrm{H}_{17} \mathrm{~N}_{3} \mathrm{O}_{3}$, the dihedral angle between the planes of the indazole ring system [maximum deviation $=0.012$ (1) $\AA$ ] and the pyran-2,4-dione ring is $54.03(6)^{\circ}$. An intramolecular $\mathrm{N}-\mathrm{H} \cdots \mathrm{O}$ hydrogen bond closes an $S(6)$ ring. The same $\mathrm{H}$ atom also participates in an intermolecular $\mathrm{N}-\mathrm{H}$. . O hydrogen bond, which generates an inversion dimer. The dimers are linked by weak $\mathrm{C}-\mathrm{H} \cdots \mathrm{O}$ contacts, thereby forming a three-dimensional network.

Keywords: crystal structure; pyran-2,4-dione; pharmacological activity; indazole derivatives.

CCDC reference: 1033266

\section{Related literature}

For pharmacological activities of indazole derivatives, see: Cerecetto et al. (2005); Jennings \& Tennant (2007); Sun et al. (1997). For innovative methods in their synthesis, see: Paul et al. (2014).

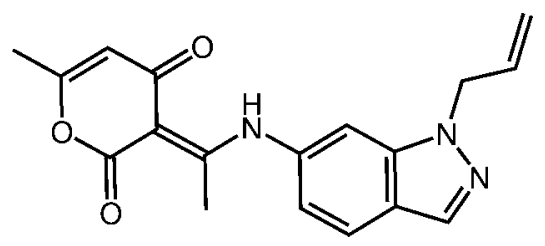

\section{Experimental}

2.1. Crystal data

$\mathrm{C}_{18} \mathrm{H}_{17} \mathrm{~N}_{3} \mathrm{O}_{3} \quad M_{r}=323.35$
Triclinic, $P \overline{1}$

$a=6.7708(11) \AA$

$b=10.5761(17) \AA$

$c=11.9643(17) \AA$

$\alpha=88.239(9)^{\circ}$

$\beta=81.123(9)^{\circ}$

$\gamma=79.140(9)^{\circ}$

\subsection{Data collection \\ Bruker X8 APEX CCD diffractometer (SADABS; Bruker, 2009) $T_{\min }=0.685, T_{\max }=0.746$ \\ 2.3. Refinement \\ $R\left[F^{2}>2 \sigma\left(F^{2}\right)\right]=0.044$ \\ $w R\left(F^{2}\right)=0.131$ \\ $S=1.04$ \\ 3929 reflections}

Absorption correction: multi-scan

$V=831.3(2) \AA^{3}$

$Z=2$

Mo $K \alpha$ radiation

$\mu=0.09 \mathrm{~mm}^{-1}$

$T=296 \mathrm{~K}$

$0.39 \times 0.34 \times 0.29 \mathrm{~mm}$

11752 measured reflections 3929 independent reflections 2930 reflections with $I>2 \sigma(I)$ $R_{\text {int }}=0.027$

Table 1

Hydrogen-bond geometry $\left(\AA,^{\circ}\right)$.

\begin{tabular}{lllll}
\hline$D-\mathrm{H} \cdots A$ & $D-\mathrm{H}$ & $\mathrm{H} \cdots A$ & $D \cdots A$ & $D-\mathrm{H} \cdots A$ \\
\hline $\mathrm{N} 3-\mathrm{H} 3 N \cdots \mathrm{O} 3$ & 0.91 & 1.77 & $2.5567(15)$ & 143 \\
$\mathrm{~N}^{2}-\mathrm{H} 3 N \cdots \mathrm{O} 3^{\mathrm{i}}$ & 0.91 & 2.51 & $3.0612(16)$ & 120 \\
$\mathrm{C} 6-\mathrm{H} 6 \cdots{ }^{\text {ii }}$ & 0.93 & 2.54 & $3.3554(19)$ & 146 \\
$\mathrm{C} 8-\mathrm{H} 8 A \cdots \mathrm{O} 1^{\mathrm{ii}}$ & 0.97 & 2.53 & $3.495(2)$ & 173 \\
\hline
\end{tabular}

Symmetry codes: (i) $-x+1,-y,-z+1$; (ii) $-x+1,-y+1,-z+1$.

Data collection: APEX2 (Bruker, 2009); cell refinement: SAINT (Bruker, 2009); data reduction: $S A I N T$; $\operatorname{program}(\mathrm{s})$ used to solve structure: SHELXS97 (Sheldrick, 2008); program(s) used to refine structure: SHELXL97 (Sheldrick, 2008); molecular graphics: ORTEP-3 for Windows (Farrugia, 2012); software used to prepare material for publication: PLATON (Spek, 2009) and publCIF (Westrip, 2010).

\section{Acknowledgements}

The authors thank the Unit of Support for Technical and Scientific Research (UATRS, CNRST) for the X-ray measurements and the University Sultan Moulay Slimane, Beni-Mellal, Morocco, for financial support.

Supporting information for this paper is available from the IUCr electronic archives (Reference: HB7311).

\section{References}

Bruker (2009). APEX2, SAINT and SADABS. Bruker AXS Inc., Madison, Wisconsin, USA.

Cerecetto, H., Gerpe, A., González, M., Arán, V. J. \& de Ocáriz, C. O. (2005). Mini Rev. Med. Chem. 5, 869-878.

Farrugia, L. J. (2012). J. Appl. Cryst. 45, 849-854.

Jennings, A. \& Tennant, M. (2007). J. Chem. Inf. Model. 47, 1829-1838.

Paul, S., Panda, S. \& Manna, D. (2014). Tetrahedron Lett. 55, 2480-2483.

Sheldrick, G. M. (2008). Acta Cryst. A64, 112-122.

Spek, A. L. (2009). Acta Cryst. D65, 148-155.

Sun, J. H., Teleha, C. A., Yan, J. S., Rodgers, J. D. \& Nugiel, D. A. (1997). J. Org. Chem. 62, 5627-5629.

Westrip, S. P. (2010). J. Appl. Cryst. 43, 920-925. 


\section{supporting information}

Acta Cryst. (2014). E70, o1256 [doi:10.1107/S1600536814024520]

\section{Crystal structure of 3-\{1-[(1-allyl-1H-indazol-6-yl)amino]ethylidene\}-6- methyl-2H-pyran-2,4(3H)-dione}

\section{Mohamed El Ghozlani, El Mostapha Rakib, Ahmed Gamouh, Mohamed Saadi and Lahcen El Ammari}

\section{S1. Comment}

Pharmacologically active indazole and its derivatives are widely used as drug in treating various human diseases including cancer, inflammation, cardiovascular, and others (Cerecetto, et al., 2005; Jennings \& Tennant, 2007; Sun, et al., 1997). This has incited researchers to develop innovative methods in their synthesis (Paul, et al., 2014).

The two fused five- and six-membered rings (N1/N2/C1 to C7), part of the molecule of the title compound, are almost planar, with the maximum deviation of 0.012 (1) $\AA$ arising from atom N1. The fused rings system is nearly perpendicular to the allyl group (C8 C9 C10) as shown in Fig. 1 (torsion angle C9 C8 N1 C7 $\left.=88.9(2)^{\circ}\right)$. Moreover, the dihedral angle between the indazole system and the plan through the atoms forming the pyran-2 ring (C12 to $\mathrm{C} 160)$ is $54.03(6)^{\circ}$.

In the crystal, molecules are connected through $\mathrm{N}-\mathrm{H} \cdots \mathrm{O}$ hydrogen bonds in the way to build dimers which are linked by weak $\mathrm{C}-\mathrm{H} \cdots \mathrm{O}$ contacts, forming a three-dimensional network as shown in Fig.2 and Table 2.

\section{S2. Experimental}

1-Allyl-6-nitroindazole (1.0 mmol) was added to a mixture of indium powder $(450 \mathrm{mg}, 3.91 \mathrm{mmol})$, and acetic acid (1.12 $\mathrm{ml}, 10 \mathrm{mmol})$ in THF $(2 \mathrm{ml})$, followed by the addition of 4-hydroxy-6-methyl-2-pyrone $(1.0 \mathrm{mmol})$ in THF ( $3 \mathrm{ml})$. The reaction mixture was stirred at $353 \mathrm{~K}$. After the reaction was completed, the reaction mixture was diluted with ethyl acetate $(30 \mathrm{ml})$, filtered through Celite, poured into $10 \% \mathrm{~K}_{2} \mathrm{CO}_{3}(25 \mathrm{ml})$, and then extracted with ethyl acetate $(30 \mathrm{ml} \times 3)$. The combined organic extracts were dried over $\mathrm{MgSO}_{4}$, filtered, and concentrated. The resulting residue was purified by flash chromatography (eluted with Ethyl acetate: Hexane 4:6). The title compound was recrystallized from the solvent mixture ethyl acetate/hexane to yield yellow blocks (yield: 85\%, m.p.: $392 \mathrm{~K}$ ).

\section{S3. Refinement}

$\mathrm{H}$ atoms were located in a difference map and treated as riding with $\mathrm{C}-\mathrm{H}=0.96 \AA, \mathrm{C}-\mathrm{H}=0.97 \AA, \mathrm{C}-\mathrm{H}=0.93 \AA$, and $\mathrm{N}-$ $\mathrm{H}=0.90 \AA$ for methyl, methylene, aromatic $\mathrm{CH}$ and $\mathrm{NH}$ respectively. All hydrogen with $U_{\text {iso }}(\mathrm{H})=1.2 U_{\text {eq }}$ (methylene,aromatic, $\mathrm{NH})$ and $U_{\text {iso }}(\mathrm{H})=1.5 U_{\text {eq }}$ for methyl. 


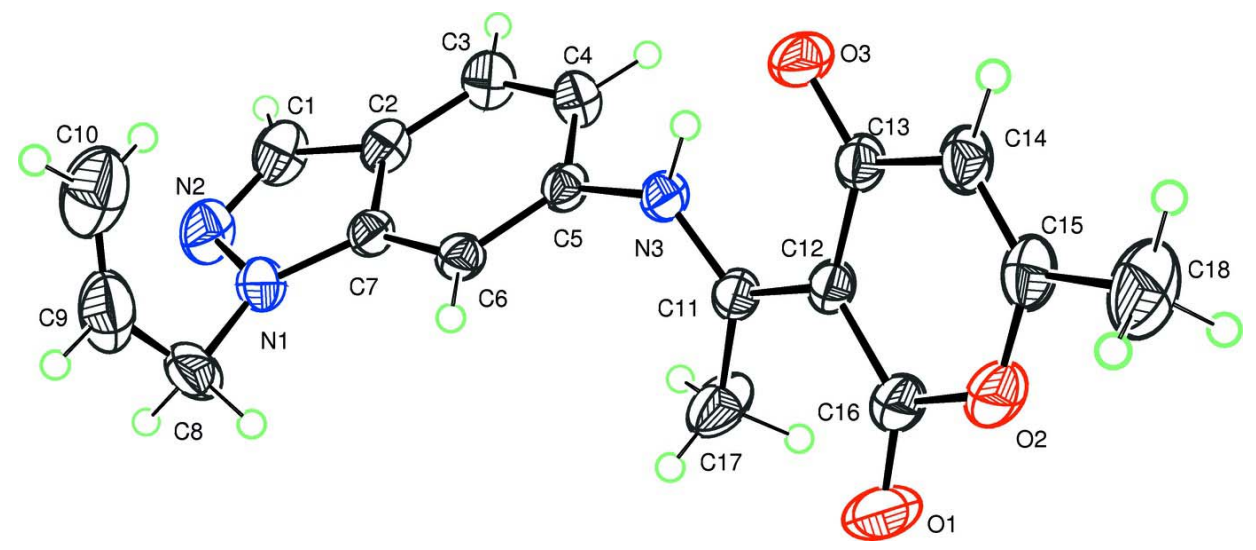

\section{Figure 1}

Molecular structure of the title compound with displacement ellipsoids drawn at the 50\% probability level.

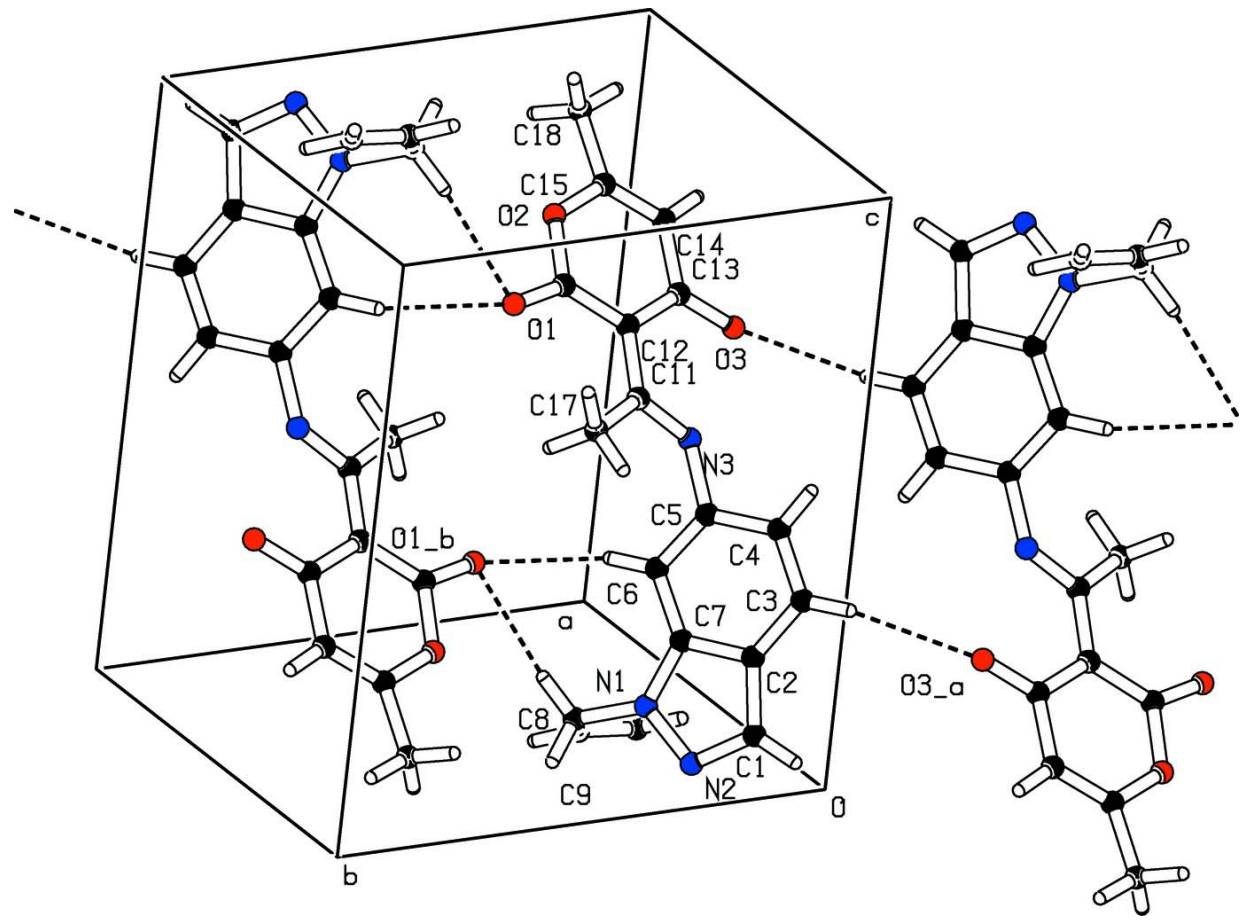

\section{Figure 2}

Partial crystal packing for the title compound showing $\mathrm{N} 3-\mathrm{H} 3 \mathrm{~N} \cdots \mathrm{O} 3, \mathrm{C} 6-\mathrm{H} 6 \cdots \mathrm{O} 1$ and $\mathrm{C} 8-\mathrm{H} 8 \mathrm{~A} \cdots \mathrm{O} 1$ hydrogen bonds as dashed lines.

\section{3-\{1-[(1-Allyl-1H-indazol-6-yl)amino]ethylidene\}-6-methyl-2H-pyran-2,4(3H)-dione}

\section{Crystal data}

$\mathrm{C}_{18} \mathrm{H}_{17} \mathrm{~N}_{3} \mathrm{O}_{3}$

$M_{r}=323.35$

Triclinic, $P \overline{1}$

Hall symbol: -P 1

$a=6.7708$ (11) $\AA$

$b=10.5761$ (17) $\AA$

$c=11.9643$ (17) $\AA$

$$
\begin{aligned}
& \alpha=88.239(9)^{\circ} \\
& \beta=81.123(9)^{\circ} \\
& \gamma=79.140(9)^{\circ} \\
& V=831.3(2) \AA^{3} \\
& Z=2 \\
& F(000)=340 \\
& D_{\mathrm{x}}=1.292 \mathrm{Mg} \mathrm{m}^{-3}
\end{aligned}
$$


Melting point: $392 \mathrm{~K}$

Mo $K \alpha$ radiation, $\lambda=0.71073 \AA$

Cell parameters from 3929 reflections

$\theta=2.6-27.9^{\circ}$

\section{Data collection}

Bruker X8 APEX CCD

diffractometer

Radiation source: fine-focus sealed tube

Graphite monochromator

$\varphi$ and $\omega$ scans

Absorption correction: multi-scan

(SADABS; Bruker, 2009)

$T_{\min }=0.685, T_{\max }=0.746$

\section{Refinement}

Refinement on $F^{2}$

Least-squares matrix: full

$R\left[F^{2}>2 \sigma\left(F^{2}\right)\right]=0.044$

$w R\left(F^{2}\right)=0.131$

$S=1.04$

3929 reflections

217 parameters

0 restraints

Primary atom site location: structure-invariant direct methods $\mu=0.09 \mathrm{~mm}^{-1}$

$T=296 \mathrm{~K}$

Block, yellow

$0.39 \times 0.34 \times 0.29 \mathrm{~mm}$

11752 measured reflections

3929 independent reflections

2930 reflections with $I>2 \sigma(I)$

$R_{\text {int }}=0.027$

$\theta_{\max }=27.9^{\circ}, \theta_{\min }=2.6^{\circ}$

$h=-8 \rightarrow 8$

$k=-12 \rightarrow 13$

$l=-15 \rightarrow 15$

Secondary atom site location: difference Fourier map

Hydrogen site location: inferred from neighbouring sites

$\mathrm{H}$-atom parameters constrained

$w=1 /\left[\sigma^{2}\left(F_{\mathrm{o}}^{2}\right)+(0.0634 P)^{2}+0.1441 P\right]$

where $P=\left(F_{\mathrm{o}}^{2}+2 F_{\mathrm{c}}^{2}\right) / 3$

$(\Delta / \sigma)_{\max }<0.001$

$\Delta \rho_{\max }=0.19 \mathrm{e} \AA^{-3}$

$\Delta \rho_{\min }=-0.19$ e $\AA^{-3}$

Special details

Geometry. All e.s.d.'s (except the e.s.d. in the dihedral angle between two 1.s. planes) are estimated using the full covariance matrix. The cell e.s.d.'s are taken into account individually in the estimation of e.s.d.'s in distances, angles and torsion angles; correlations between e.s.d.'s in cell parameters are only used when they are defined by crystal symmetry. An approximate (isotropic) treatment of cell e.s.d.'s is used for estimating e.s.d.'s involving 1.s. planes.

Refinement. Refinement of $F^{2}$ against all reflections. The weighted $R$-factor $w R$ and goodness of fit $S$ are based on $F^{2}$, conventional $R$-factors $R$ are based on $F$, with $F$ set to zero for negative $F^{2}$. The threshold expression of $F^{2}>\sigma\left(F^{2}\right)$ is used only for calculating $R$-factors(gt) $e t c$. and is not relevant to the choice of reflections for refinement. $R$-factors based on $F^{2}$ are statistically about twice as large as those based on $F$, and $R$ - factors based on all data will be even larger.

Fractional atomic coordinates and isotropic or equivalent isotropic displacement parameters $\left(\AA^{2}\right)$

\begin{tabular}{lllll}
\hline & $x$ & $y$ & $z$ & $U_{\text {iss }} / U_{\text {eq }}$ \\
\hline C1 & $-0.2126(2)$ & $0.27561(17)$ & $0.19077(14)$ & $0.0535(4)$ \\
H1 & -0.3315 & 0.2495 & 0.1792 & $0.064^{*}$ \\
C2 & $-0.1187(2)$ & $0.24555(13)$ & $0.28909(11)$ & $0.0394(3)$ \\
C3 & $-0.1571(2)$ & $0.17674(15)$ & $0.38933(12)$ & $0.0453(4)$ \\
H3 & -0.2704 & 0.1375 & 0.4040 & $0.054^{*}$ \\
C4 & $-0.0230(2)$ & $0.16880(14)$ & $0.46546(11)$ & $0.0419(3)$ \\
H4 & -0.0454 & 0.1230 & 0.5324 & $0.050^{*}$ \\
C5 & $0.14799(19)$ & $0.22876(12)$ & $0.44391(10)$ & $0.0327(3)$ \\
C6 & $0.1916(2)$ & $0.29707(12)$ & $0.34631(10)$ & $0.0347(3)$ \\
H6 & 0.3054 & 0.3358 & 0.3323 & $0.042^{*}$ \\
C7 & $0.0533(2)$ & $0.30446(12)$ & $0.26930(10)$ & $0.0352(3)$ \\
C8 & $0.2094(3)$ & $0.42381(18)$ & $0.10121(14)$ & $0.0664(5)$
\end{tabular}




$\begin{array}{lllll}\text { H8A } & 0.2818 & 0.4594 & 0.1529 & 0.080^{*} \\ \text { H8B } & 0.1464 & 0.4943 & 0.0572 & 0.080^{*} \\ \text { C9 } & 0.3587(4) & 0.3318(3) & 0.02309(16) & 0.0820(7) \\ \text { H9 } & 0.4607 & 0.3652 & -0.0227 & 0.098^{*} \\ \text { C10 } & 0.3598(3) & 0.2112(3) & 0.01307(18) & 0.0866(7) \\ \text { H10A } & 0.2609 & 0.1733 & 0.0570 & 0.104^{*} \\ \text { H10B } & 0.4595 & 0.1616 & -0.0382 & 0.104^{*} \\ \text { C11 } & 0.3460(2) & 0.29146(12) & 0.58397(10) & 0.0349(3) \\ \text { C12 } & 0.4993(2) & 0.24657(12) & 0.65330(10) & 0.0341(3) \\ \text { C13 } & 0.5675(2) & 0.11088(13) & 0.66967(10) & 0.0359(3) \\ \text { C14 } & 0.7230(2) & 0.07406(14) & 0.74087(11) & 0.0429(3) \\ \text { H14 } & 0.7646 & -0.0126 & 0.7568 & 0.051^{*} \\ \text { C15 } & 0.8078(2) & 0.16072(16) & 0.78399(12) & 0.0474(4) \\ \text { C16 } & 0.5936(3) & 0.33761(15) & 0.70210(12) & 0.0482(4) \\ \text { C17 } & 0.2422(3) & 0.42837(15) & 0.57937(16) & 0.0671(6) \\ \text { H17A } & 0.1140 & 0.4326 & 0.5532 & 0.101^{*} \\ \text { H17B } & 0.3260 & 0.4753 & 0.5284 & 0.101^{*} \\ \text { H17C } & 0.2197 & 0.4655 & 0.6535 & 0.101^{*} \\ \text { C18 } & 0.9761(3) & 0.1362(2) & 0.85373(18) & 0.0806(6) \\ \text { H18A } & 0.9316 & 0.1796 & 0.9250 & 0.121^{*} \\ \text { H18C } & 1.0918 & 0.1679 & 0.8145 & 0.121^{*} \\ \text { H18B } & 1.0133 & 0.0454 & 0.8665 & 0.121^{*} \\ \text { N1 } & 0.0511(2) & 0.36448(12) & 0.16653(10) & 0.0472(3) \\ \text { N2 } & -0.1112(2) & 0.34456(14) & 0.11844(11) & 0.0574(4) \\ \text { N3 } & 0.28794(17) & 0.20639(10) & 0.52426(9) & 0.0350(3) \\ \text { H3N } & 0.3443 & 0.1235 & 0.5379 & 0.042^{*} \\ \text { O1 } & 0.5633(3) & 0.45366(11) & 0.69316(13) & 0.0832(5) \\ \text { O2 } & 0.74794(18) & 0.28942(11) & 0.76619(9) & 0.0567(3) \\ \text { O3 } & 0.50060(18) & 0.02513(9) & 0.62444(9) & 0.0538(3) \\ & & & & \end{array}$

Atomic displacement parameters $\left(\AA^{2}\right)$

\begin{tabular}{lllllll}
\hline & $U^{11}$ & $U^{22}$ & $U^{33}$ & $U^{12}$ & $U^{13}$ & $U^{23}$ \\
\hline C1 & $0.0429(9)$ & $0.0691(11)$ & $0.0520(9)$ & $-0.0058(8)$ & $-0.0233(7)$ & $-0.0044(8)$ \\
C2 & $0.0330(7)$ & $0.0437(8)$ & $0.0413(7)$ & $-0.0003(6)$ & $-0.0124(5)$ & $-0.0057(6)$ \\
C3 & $0.0325(7)$ & $0.0595(9)$ & $0.0459(8)$ & $-0.0136(6)$ & $-0.0057(6)$ & $-0.0009(7)$ \\
C4 & $0.0404(8)$ & $0.0494(8)$ & $0.0360(7)$ & $-0.0096(6)$ & $-0.0055(6)$ & $0.0045(6)$ \\
C5 & $0.0351(7)$ & $0.0320(6)$ & $0.0313(6)$ & $-0.0016(5)$ & $-0.0108(5)$ & $-0.0025(5)$ \\
C6 & $0.0392(7)$ & $0.0317(6)$ & $0.0362(6)$ & $-0.0083(5)$ & $-0.0128(5)$ & $0.0005(5)$ \\
C7 & $0.0413(7)$ & $0.0306(6)$ & $0.0340(6)$ & $-0.0015(5)$ & $-0.0130(5)$ & $-0.0012(5)$ \\
C8 & $0.1033(15)$ & $0.0663(11)$ & $0.0490(9)$ & $-0.0470(11)$ & $-0.0384(10)$ & $0.0265(8)$ \\
C9 & $0.0885(15)$ & $0.130(2)$ & $0.0434(9)$ & $-0.0647(15)$ & $-0.0069(10)$ & $0.0047(11)$ \\
C10 & $0.0708(14)$ & $0.125(2)$ & $0.0651(12)$ & $-0.0253(14)$ & $0.0001(10)$ & $-0.0205(13)$ \\
C11 & $0.0409(7)$ & $0.0328(7)$ & $0.0307(6)$ & $-0.0022(5)$ & $-0.0090(5)$ & $-0.0023(5)$ \\
C12 & $0.0401(7)$ & $0.0347(7)$ & $0.0288(6)$ & $-0.0061(5)$ & $-0.0099(5)$ & $-0.0020(5)$ \\
C13 & $0.0405(7)$ & $0.0375(7)$ & $0.0298(6)$ & $-0.0037(6)$ & $-0.0102(5)$ & $-0.0011(5)$ \\
C14 & $0.0483(8)$ & $0.0446(8)$ & $0.0344(6)$ & $0.0024(6)$ & $-0.0160(6)$ & $0.0005(6)$ \\
C15 & $0.0472(9)$ & $0.0594(9)$ & $0.0366(7)$ & $-0.0040(7)$ & $-0.0163(6)$ & $-0.0006(6)$
\end{tabular}


supporting information

\begin{tabular}{lllllll}
$\mathrm{C} 16$ & $0.0641(10)$ & $0.0441(8)$ & $0.0438(8)$ & $-0.0155(7)$ & $-0.0247(7)$ & $0.0010(6)$ \\
$\mathrm{C} 17$ & $0.0909(14)$ & $0.0388(8)$ & $0.0736(11)$ & $0.0151(8)$ & $-0.0484(10)$ & $-0.0163(8)$ \\
$\mathrm{C} 18$ & $0.0723(13)$ & $0.1046(16)$ & $0.0736(12)$ & $-0.0078(12)$ & $-0.0479(11)$ & $-0.0039(11)$ \\
$\mathrm{N} 1$ & $0.0629(8)$ & $0.0466(7)$ & $0.0385(6)$ & $-0.0134(6)$ & $-0.0258(6)$ & $0.0080(5)$ \\
$\mathrm{N} 2$ & $0.0622(9)$ & $0.0659(9)$ & $0.0493(7)$ & $-0.0067(7)$ & $-0.0324(7)$ & $0.0040(6)$ \\
$\mathrm{N} 3$ & $0.0408(6)$ & $0.0308(5)$ & $0.0347(5)$ & $-0.0014(5)$ & $-0.0159(5)$ & $0.0006(4)$ \\
$\mathrm{O} 1$ & $0.1312(13)$ & $0.0405(7)$ & $0.1001(10)$ & $-0.0286(7)$ & $-0.0724(10)$ & $0.0073(6)$ \\
$\mathrm{O} 2$ & $0.0669(7)$ & $0.0579(7)$ & $0.0567(6)$ & $-0.0191(6)$ & $-0.0349(6)$ & $-0.0013(5)$ \\
$\mathrm{O} 3$ & $0.0747(8)$ & $0.0333(5)$ & $0.0610(7)$ & $-0.0055(5)$ & $-0.0394(6)$ & $-0.0006(4)$ \\
\hline
\end{tabular}

Geometric parameters $\left(\AA,{ }^{o}\right)$

\begin{tabular}{|c|c|c|c|}
\hline $\mathrm{C} 1-\mathrm{N} 2$ & $1.311(2)$ & $\mathrm{C} 11-\mathrm{N} 3$ & $1.3166(16)$ \\
\hline $\mathrm{C} 1-\mathrm{C} 2$ & $1.4215(19)$ & $\mathrm{C} 11-\mathrm{C} 12$ & $1.4309(18)$ \\
\hline $\mathrm{C} 1-\mathrm{H} 1$ & 0.9300 & $\mathrm{C} 11-\mathrm{C} 17$ & $1.4890(19)$ \\
\hline $\mathrm{C} 2-\mathrm{C} 3$ & $1.399(2)$ & $\mathrm{C} 12-\mathrm{C} 16$ & $1.4339(18)$ \\
\hline $\mathrm{C} 2-\mathrm{C} 7$ & $1.407(2)$ & $\mathrm{C} 12-\mathrm{C} 13$ & $1.4394(18)$ \\
\hline $\mathrm{C} 3-\mathrm{C} 4$ & $1.3723(19)$ & $\mathrm{C} 13-\mathrm{O} 3$ & $1.2575(16)$ \\
\hline $\mathrm{C} 3-\mathrm{H} 3$ & 0.9300 & $\mathrm{C} 13-\mathrm{C} 14$ & $1.4447(18)$ \\
\hline $\mathrm{C} 4-\mathrm{C} 5$ & $1.408(2)$ & $\mathrm{C} 14-\mathrm{C} 15$ & $1.325(2)$ \\
\hline $\mathrm{C} 4-\mathrm{H} 4$ & 0.9300 & $\mathrm{C} 14-\mathrm{H} 14$ & 0.9300 \\
\hline $\mathrm{C} 5-\mathrm{C} 6$ & $1.3765(18)$ & $\mathrm{C} 15-\mathrm{O} 2$ & $1.3649(19)$ \\
\hline $\mathrm{C} 5-\mathrm{N} 3$ & $1.4355(15)$ & $\mathrm{C} 15-\mathrm{C} 18$ & $1.494(2)$ \\
\hline $\mathrm{C} 6-\mathrm{C} 7$ & $1.4022(17)$ & $\mathrm{C} 16-\mathrm{O} 1$ & $1.2099(19)$ \\
\hline $\mathrm{C} 6-\mathrm{H} 6$ & 0.9300 & $\mathrm{C} 16-\mathrm{O} 2$ & $1.4010(18)$ \\
\hline $\mathrm{C} 7-\mathrm{N} 1$ & $1.3672(17)$ & $\mathrm{C} 17-\mathrm{H} 17 \mathrm{~A}$ & 0.9600 \\
\hline $\mathrm{C} 8-\mathrm{N} 1$ & $1.456(2)$ & C17-H17B & 0.9600 \\
\hline $\mathrm{C} 8-\mathrm{C} 9$ & $1.495(3)$ & $\mathrm{C} 17-\mathrm{H} 17 \mathrm{C}$ & 0.9600 \\
\hline $\mathrm{C} 8-\mathrm{H} 8 \mathrm{~A}$ & 0.9700 & $\mathrm{C} 18-\mathrm{H} 18 \mathrm{~A}$ & 0.9600 \\
\hline $\mathrm{C} 8-\mathrm{H} 8 \mathrm{~B}$ & 0.9700 & $\mathrm{C} 18-\mathrm{H} 18 \mathrm{C}$ & 0.9600 \\
\hline $\mathrm{C} 9-\mathrm{C} 10$ & $1.284(3)$ & C18-H18B & 0.9600 \\
\hline C9-H9 & 0.9300 & $\mathrm{~N} 1-\mathrm{N} 2$ & $1.3684(18)$ \\
\hline $\mathrm{C} 10-\mathrm{H} 10 \mathrm{~A}$ & 0.9300 & $\mathrm{~N} 3-\mathrm{H} 3 \mathrm{~N}$ & 0.9090 \\
\hline $\mathrm{C} 10-\mathrm{H} 10 \mathrm{~B}$ & 0.9300 & & \\
\hline $\mathrm{N} 2-\mathrm{C} 1-\mathrm{C} 2$ & $111.83(14)$ & $\mathrm{C} 11-\mathrm{C} 12-\mathrm{C} 16$ & $119.56(12)$ \\
\hline $\mathrm{N} 2-\mathrm{C} 1-\mathrm{H} 1$ & 124.1 & $\mathrm{C} 11-\mathrm{C} 12-\mathrm{C} 13$ & $120.72(11)$ \\
\hline $\mathrm{C} 2-\mathrm{C} 1-\mathrm{H} 1$ & 124.1 & $\mathrm{C} 16-\mathrm{C} 12-\mathrm{C} 13$ & $119.64(12)$ \\
\hline $\mathrm{C} 3-\mathrm{C} 2-\mathrm{C} 7$ & $119.78(12)$ & $\mathrm{O} 3-\mathrm{C} 13-\mathrm{C} 12$ & $123.39(12)$ \\
\hline $\mathrm{C} 3-\mathrm{C} 2-\mathrm{C} 1$ & $136.29(14)$ & $\mathrm{O} 3-\mathrm{C} 13-\mathrm{C} 14$ & $119.55(12)$ \\
\hline $\mathrm{C} 7-\mathrm{C} 2-\mathrm{C} 1$ & $103.93(13)$ & $\mathrm{C} 12-\mathrm{C} 13-\mathrm{C} 14$ & $117.06(12)$ \\
\hline $\mathrm{C} 4-\mathrm{C} 3-\mathrm{C} 2$ & $118.11(13)$ & $\mathrm{C} 15-\mathrm{C} 14-\mathrm{C} 13$ & $121.62(13)$ \\
\hline $\mathrm{C} 4-\mathrm{C} 3-\mathrm{H} 3$ & 120.9 & $\mathrm{C} 15-\mathrm{C} 14-\mathrm{H} 14$ & 119.2 \\
\hline $\mathrm{C} 2-\mathrm{C} 3-\mathrm{H} 3$ & 120.9 & $\mathrm{C} 13-\mathrm{C} 14-\mathrm{H} 14$ & 119.2 \\
\hline $\mathrm{C} 3-\mathrm{C} 4-\mathrm{C} 5$ & $121.11(13)$ & $\mathrm{C} 14-\mathrm{C} 15-\mathrm{O} 2$ & $121.58(13)$ \\
\hline $\mathrm{C} 3-\mathrm{C} 4-\mathrm{H} 4$ & 119.4 & $\mathrm{C} 14-\mathrm{C} 15-\mathrm{C} 18$ & $127.34(16)$ \\
\hline $\mathrm{C} 5-\mathrm{C} 4-\mathrm{H} 4$ & 119.4 & $\mathrm{O} 2-\mathrm{C} 15-\mathrm{C} 18$ & $111.07(14)$ \\
\hline $\mathrm{C} 6-\mathrm{C} 5-\mathrm{C} 4$ & $122.61(12)$ & $\mathrm{O} 1-\mathrm{C} 16-\mathrm{O} 2$ & $113.34(13)$ \\
\hline
\end{tabular}


supporting information

$\begin{array}{llll}\text { C6-C5-N3 } & 120.24(12) & \mathrm{O} 1-\mathrm{C} 16-\mathrm{C} 12 & 128.87(14) \\ \mathrm{C} 4-\mathrm{C} 5-\mathrm{N} 3 & 116.93(11) & \mathrm{O} 2-\mathrm{C} 16-\mathrm{C} 12 & 117.75(13) \\ \mathrm{C} 5-\mathrm{C} 6-\mathrm{C} 7 & 115.68(12) & \mathrm{C} 11-\mathrm{C} 17-\mathrm{H} 17 \mathrm{~A} & 109.5 \\ \mathrm{C} 5-\mathrm{C} 6-\mathrm{H} 6 & 122.2 & \mathrm{C} 11-\mathrm{C} 17-\mathrm{H} 17 \mathrm{~B} & 109.5 \\ \mathrm{C} 7-\mathrm{C} 6-\mathrm{H} 6 & 122.2 & \mathrm{H} 17 \mathrm{~A}-\mathrm{C} 17-\mathrm{H} 17 \mathrm{~B} & 109.5 \\ \mathrm{~N} 1-\mathrm{C} 7-\mathrm{C} 6 & 130.43(13) & \mathrm{C} 11-\mathrm{C} 17-\mathrm{H} 17 \mathrm{C} & 109.5 \\ \mathrm{~N} 1-\mathrm{C} 7-\mathrm{C} 2 & 106.87(12) & \mathrm{H} 17 \mathrm{~A}-\mathrm{C} 17-\mathrm{H} 17 \mathrm{C} & 109.5 \\ \mathrm{C} 6-\mathrm{C} 7-\mathrm{C} 2 & 122.70(12) & \mathrm{H} 17 \mathrm{~B}-\mathrm{C} 17-\mathrm{H} 17 \mathrm{C} & 109.5 \\ \mathrm{~N} 1-\mathrm{C} 8-\mathrm{C} 9 & 113.14(15) & \mathrm{C} 15-\mathrm{C} 18-\mathrm{H} 18 \mathrm{~A} & 109.5 \\ \mathrm{~N} 1-\mathrm{C} 8-\mathrm{H} 8 \mathrm{~A} & 109.0 & \mathrm{C} 15-\mathrm{C} 18-\mathrm{H} 18 \mathrm{C} & 109.5 \\ \mathrm{C} 9-\mathrm{C} 8-\mathrm{H} 8 \mathrm{~A} & 109.0 & \mathrm{H} 18 \mathrm{~A}-\mathrm{C} 18-\mathrm{H} 18 \mathrm{C} & 109.5 \\ \text { N1-C8-H8B } & 109.0 & \mathrm{C} 15-\mathrm{C} 18-\mathrm{H} 18 \mathrm{~B} & 109.5 \\ \mathrm{C} 9-\mathrm{C} 8-\mathrm{H} 8 \mathrm{~B} & 109.0 & \mathrm{H} 18 \mathrm{~A}-\mathrm{C} 18-\mathrm{H} 18 \mathrm{~B} & 109.5 \\ \mathrm{H} 8 \mathrm{~A}-\mathrm{C} 8-\mathrm{H} 8 \mathrm{~B} & 107.8 & \mathrm{H} 18 \mathrm{C}-\mathrm{C} 18-\mathrm{H} 18 \mathrm{~B} & 109.5 \\ \mathrm{C} 10-\mathrm{C} 9-\mathrm{C} 8 & 126.33(19) & \mathrm{C} 7-\mathrm{N} 1-\mathrm{N} 2 & 110.85(13) \\ \mathrm{C} 10-\mathrm{C} 9-\mathrm{H} 9 & 116.8 & \mathrm{C} 7-\mathrm{N} 1-\mathrm{C} 8 & 128.13(13) \\ \mathrm{C} 8-\mathrm{C} 9-\mathrm{H} 9 & 116.8 & \mathrm{~N} 2-\mathrm{N} 1-\mathrm{C} 8 & 120.20(12) \\ \mathrm{C} 9-\mathrm{C} 10-\mathrm{H} 10 \mathrm{~A} & 120.0 & \mathrm{C} 1-\mathrm{N} 2-\mathrm{N} 1 & 106.50(12) \\ \mathrm{C} 9-\mathrm{C} 10-\mathrm{H} 10 \mathrm{~B} & 120.0 & \mathrm{C} 11-\mathrm{N} 3-\mathrm{C} 3 \mathrm{~N} & 113.9 \\ \mathrm{H} 10 \mathrm{~A}-\mathrm{C} 10-\mathrm{H} 10 \mathrm{~B} & 120.0 & \mathrm{C} 5-\mathrm{N} 3-\mathrm{H} 3 \mathrm{~N} & 117.7 \\ \text { N3-C11-C12 } & 118.25(11) & \mathrm{C} 15-\mathrm{O} 2-\mathrm{C} 16 & 122.25(11) \\ \text { N3-C11-C17 } & 118.15(12) & & \\ \text { C12-C11-C17 } & 123.55(12) & & \end{array}$

Hydrogen-bond geometry $\left(\AA,{ }^{\circ}\right)$

\begin{tabular}{lllll}
\hline$D-\mathrm{H} \cdots A$ & $D-\mathrm{H}$ & $\mathrm{H} \cdots A$ & $D \cdots A$ & $D-\mathrm{H} \cdots A$ \\
\hline $\mathrm{N} 3-\mathrm{H} 3 N \cdots \mathrm{O} 3$ & 0.91 & 1.77 & $2.5567(15)$ & 143 \\
$\mathrm{~N} 3-\mathrm{H} 3 N \cdots \mathrm{O} 3^{\mathrm{i}}$ & 0.91 & 2.51 & $3.0612(16)$ & 120 \\
$\mathrm{C} 6-\mathrm{H} 6 \cdots{ }^{\mathrm{ii}}$ & 0.93 & 2.54 & $3.3554(19)$ & 146 \\
$\mathrm{C} 8-\mathrm{H} 8 A \cdots \mathrm{O} 1^{\mathrm{ii}}$ & 0.97 & 2.53 & $3.495(2)$ & 173 \\
\hline
\end{tabular}

Symmetry codes: (i) $-x+1,-y,-z+1$; (ii) $-x+1,-y+1,-z+1$. 